\title{
ECONOMICS OF FISH PRODUCTION IN MARRIOTT LAKE
} Abdel-Hkim,N.F.*; A. K. Abd- Elhmied ${ }^{\star *}$ and Sh. A.Abdel-Momen ${ }^{\star *}$ Dept. of Animal Production, Faculty of Agriculture, Al-Azhar University

** Dept. of Agric. Economic production - Faculty of Agriculture-AlAzhar University

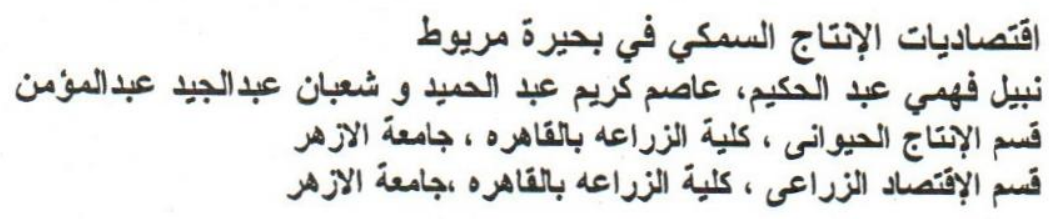

الملغص

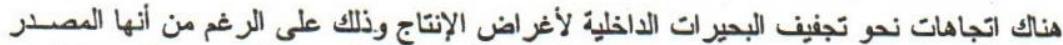

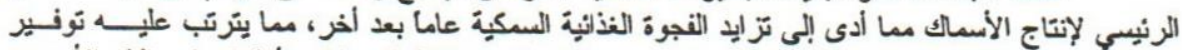

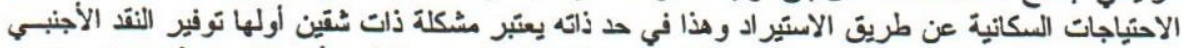

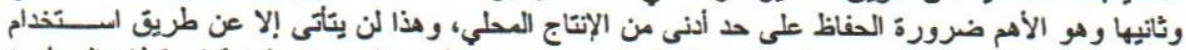

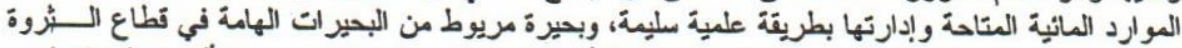

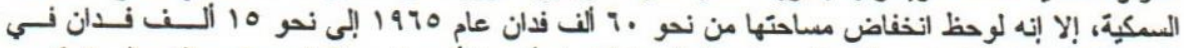

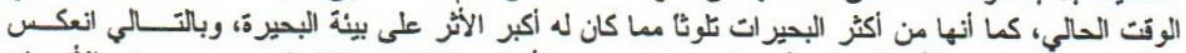

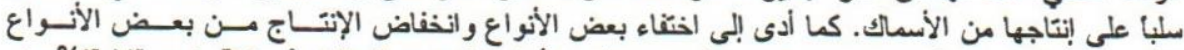

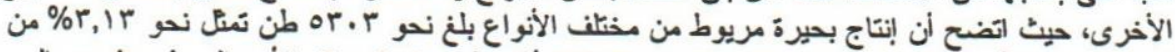

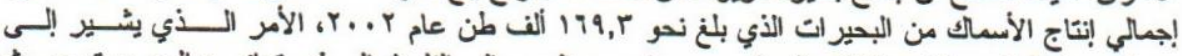

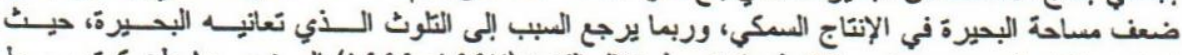

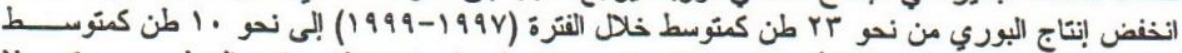

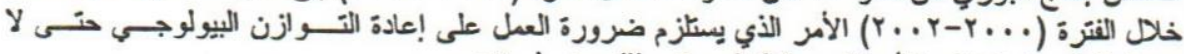

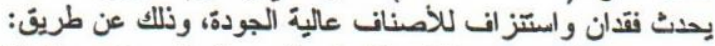

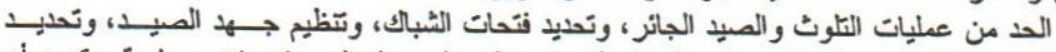

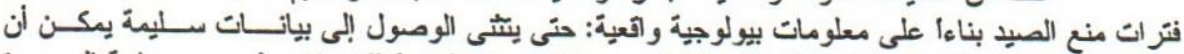

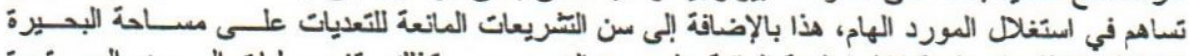

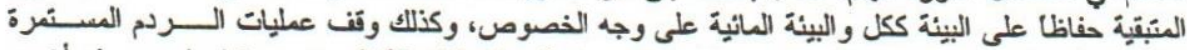

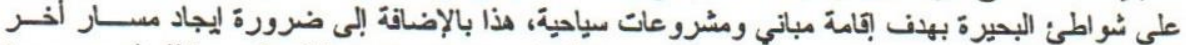

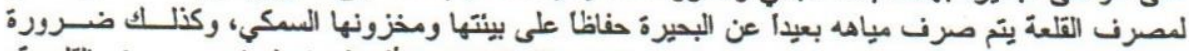

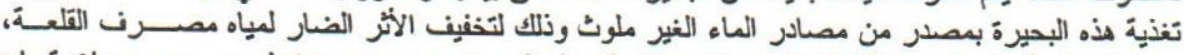

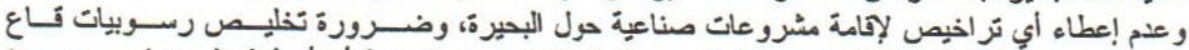

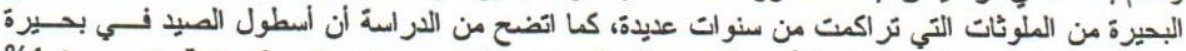

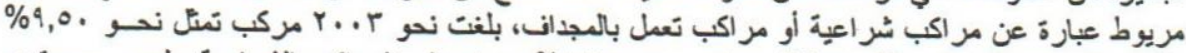

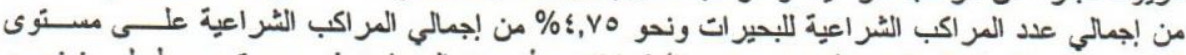

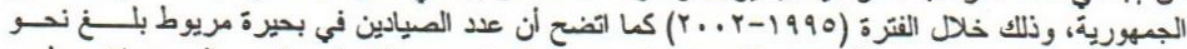

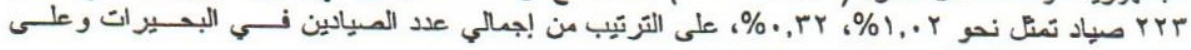
مستوى الجمهورية وذلك خلال نفس الفترة. 


\section{المقدمة}

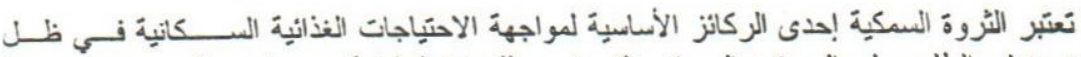

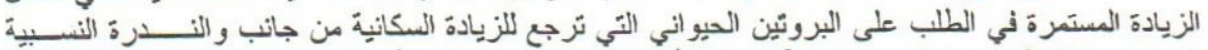

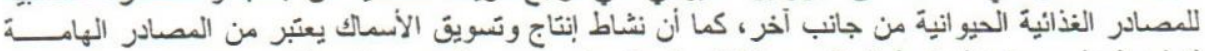

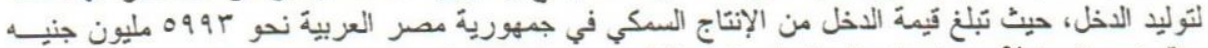

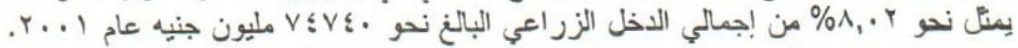

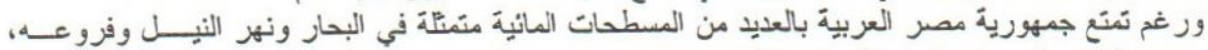

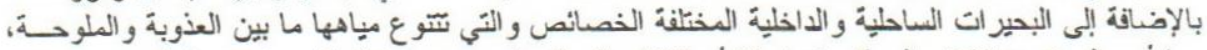

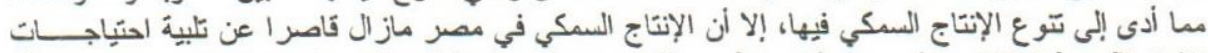

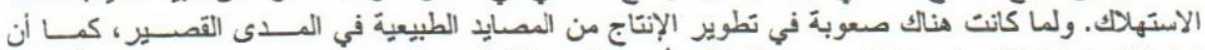

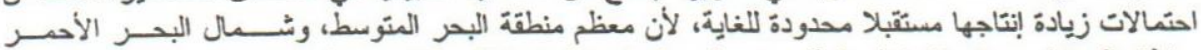

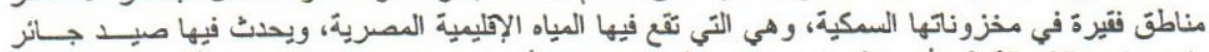

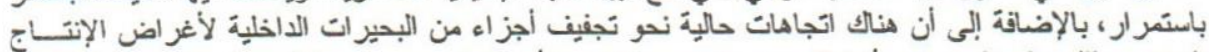

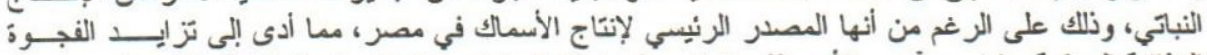

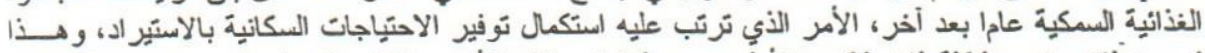

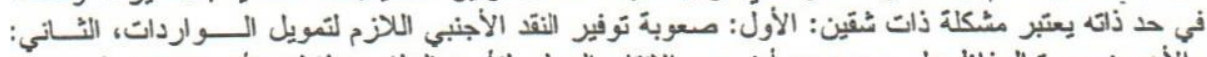

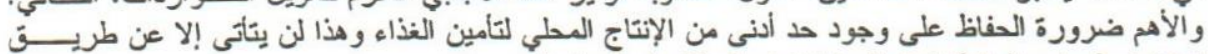

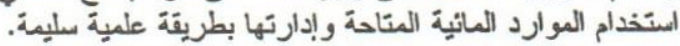

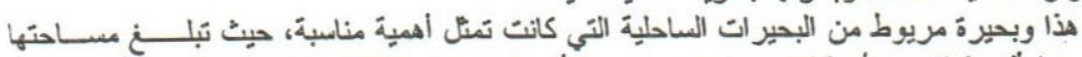

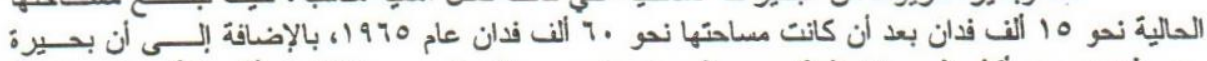

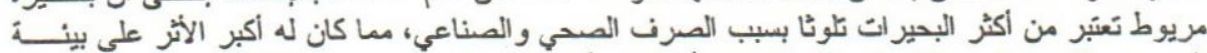

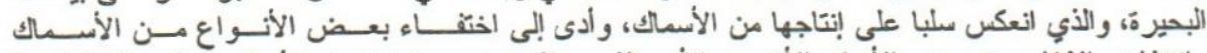

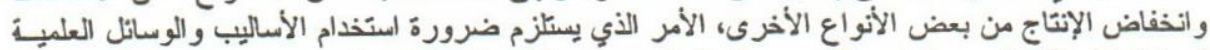

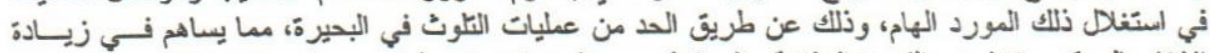

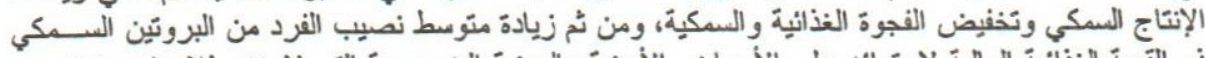

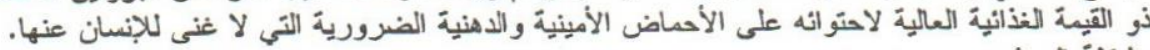

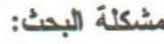

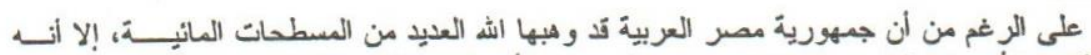

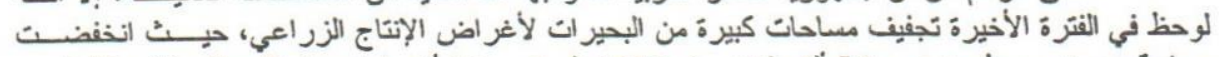

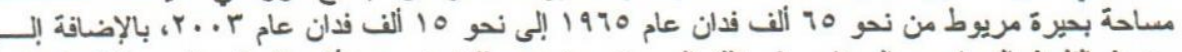

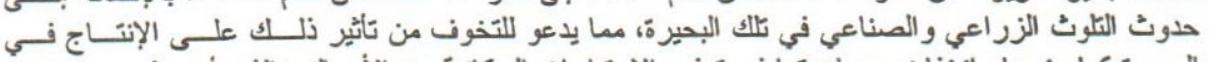

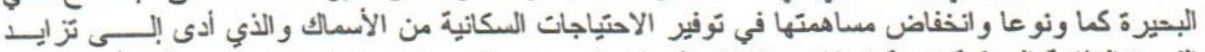

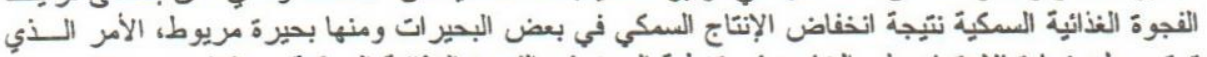

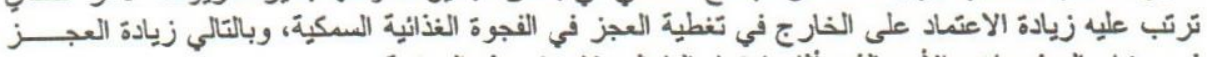

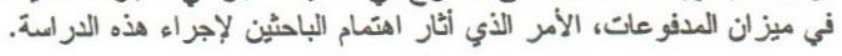

\section{هدف البحث}

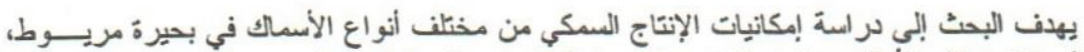

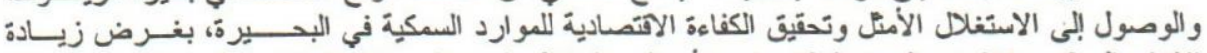

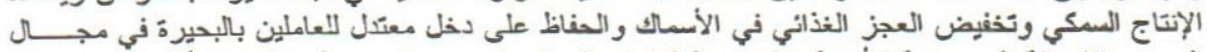

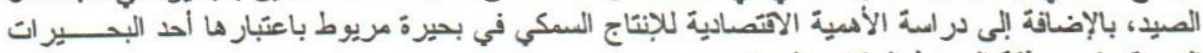

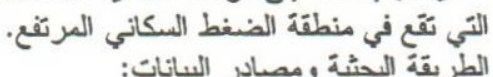

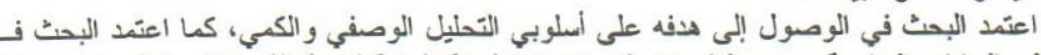

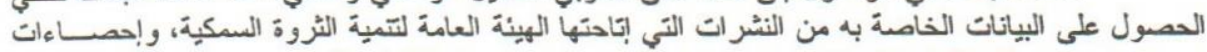

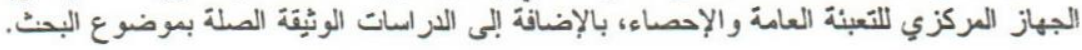




$$
\text { الموتع الجغر افي للبحيزة: }
$$

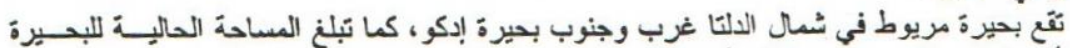

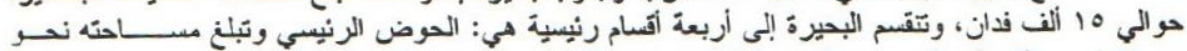

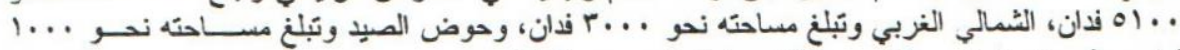

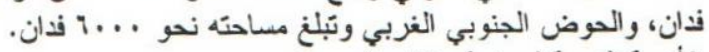
الأهمية النسبية لمساحة وإنتاج البحيرةً:

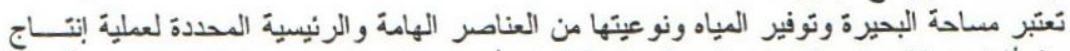

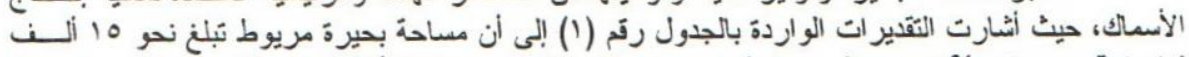

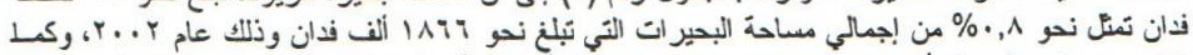

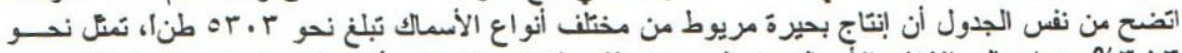

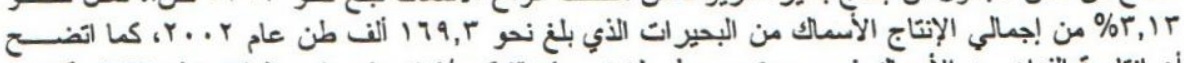

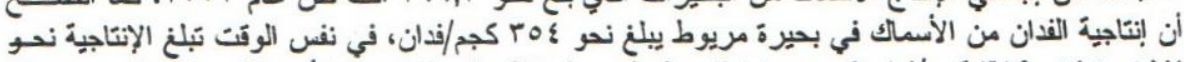

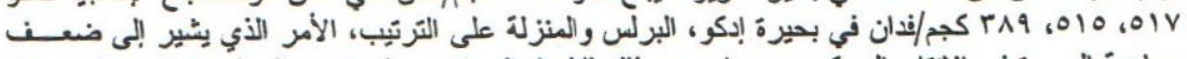

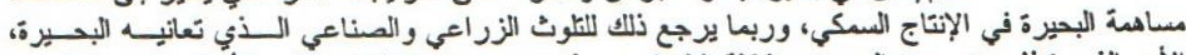

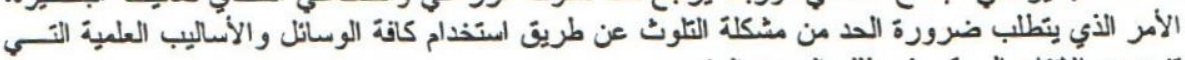

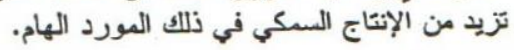

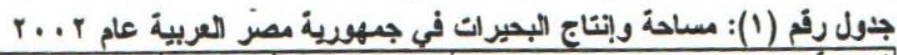

\begin{tabular}{|c|c|c|c|c|c|}
\hline | الإتاجية & \multicolumn{2}{|c|}{ الإتَاج } & \multicolumn{2}{|c|}{ السماهة } & \multirow{2}{*}{ |لبحيرة } \\
\hline كجم/فدان & $\%$ & بالطن & $\%$ & ألف ذذاني & \\
\hline reg & $r \leqslant \leqslant 9$ & 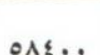 & A. \{ & 10. & |لالبحيرات الشمالية: \\
\hline 010 & $r 0, r)$ & 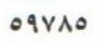 & $T, Y Y$ & 119 & ل \\
\hline 018 & י, . & ו.r & $1, \cdot v$ & r. & إبكر \\
\hline rọ & $r, 1 r$ & or.r & $\cdot, \wedge$ & 10 & مريوط \\
\hline & & & & & |لالنفنضات الساحلية: \\
\hline 19 & i,Ar & ri.. & $1,0 \mathrm{~V}$ & 19. & | البردويل \\
\hline rV &., 11 & 19. & $\cdot, \mathrm{rA}$ & $\mathrm{r}$ & ملاحة بور فواد \\
\hline זי & $1,1 \leqslant$ & 1940 & 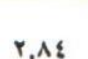 & or & البحيرات الاخلية: \\
\hline ro &., $\mathrm{vr}$ & $|r r|$ & 1,19 & ro & منخفض الريان r, \\
\hline 19 & $|r, \Lambda|$ & rerri & 17,91 & iro. & بحيرة ناصر \\
\hline १ะ & $r, r_{0}$ & . & $r, r)$ & i. & بحيرتى المزئَّ والتمساح \\
\hline & $1 \ldots$ & 17951. & $1 \ldots$ & $1 \mathrm{1AT4}$ & بطلة البحيرات \\
\hline
\end{tabular}

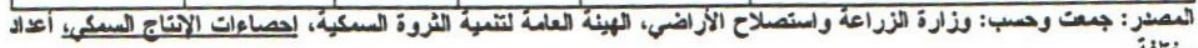

تطرد الإتناج السكي في البحيرات الشمالية حسب الأصناف:

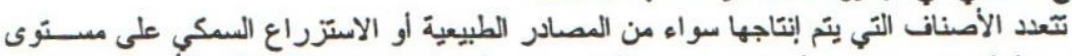

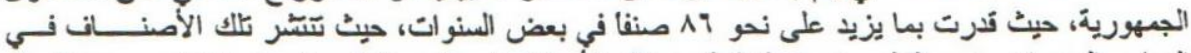

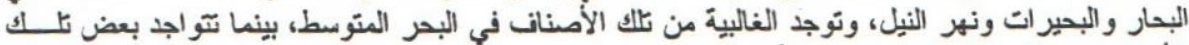

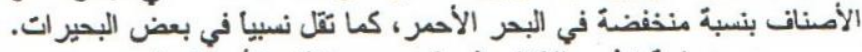

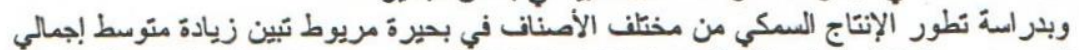

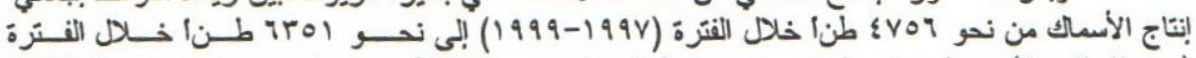

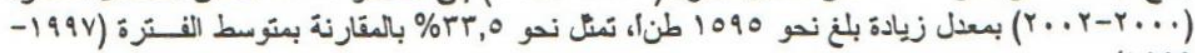
.$(1999$ كما اتضح زيادة متوسط إنتاج كل من البلطي، والقر اميط والحيتان، ومبروك الحشانش والأصنات

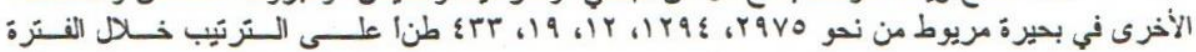




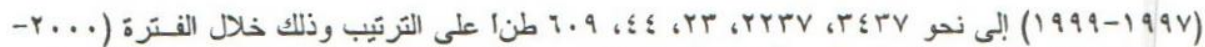

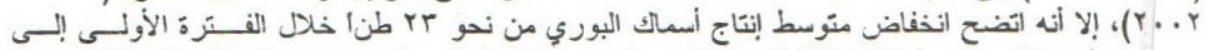

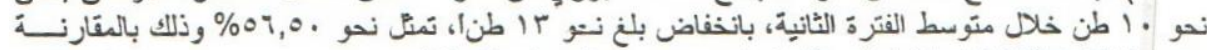

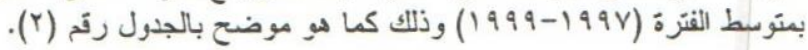

عوامل الإتتاج السمكي في بحيرة مريوط:

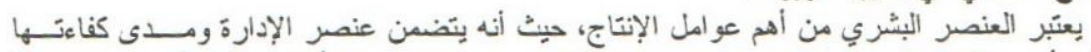

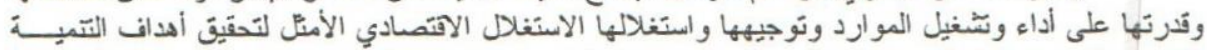

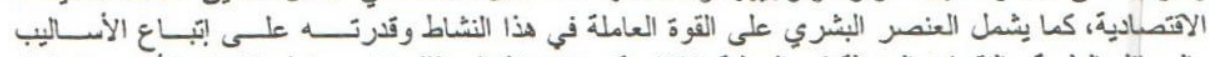

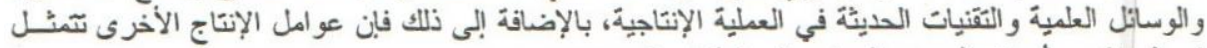

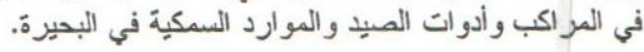

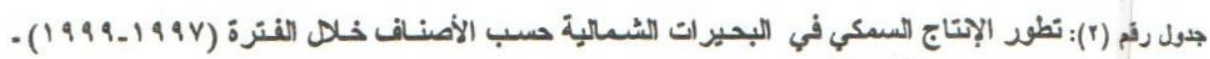

\begin{tabular}{|c|c|c|c|c|c|c|c|c|}
\hline \multicolumn{9}{|c|}{$\left(r^{2} \cdot r_{-} r^{2} \cdots\right)$} \\
\hline \multicolumn{2}{|c|}{ بحيرة إدكو } & \multicolumn{2}{|c|}{ بحيرة البرلس } & \multicolumn{2}{|c|}{ بحيرة المنزلة } & \multicolumn{2}{|c|}{ بحيرة مريوط } & \\
\hline$-r \ldots$ & -1998 & $-Y \ldots$ & -1998 & $-r \ldots$ & $1999-1998$ & $-r \ldots$ & -1998 & 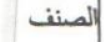 \\
\hline r...r & 1999 & r..r & 1999 & $r \ldots r$ & & $r \ldots r$ & 1999 & . \\
\hline RVTI & AIr & rinon & ro111 & $r \leqslant \div \wedge 1$ & TVATO & $r \leqslant r v$ & rqvo & \\
\hline- & - & - & - & - & - & - & - & \\
\hline or. & @० & Mrry & 1.rto & Г৭१५ & r. $\lfloor 0$ & 1. & rr & \\
\hline var & $T \leqslant$ & riq1 & rAY. & $1 . \leqslant$ १ & ؛ TrA & rYTV & Irq! & \\
\hline- & - & 1.9 & १४ & ११५ & QYY & - & - & \\
\hline- & - & 01 & 0 & $v \leqslant 1$ & 111. & - & - & \\
\hline 19 & ir & $11 v$ & Irg & $1 \leqslant 10$ & rTV & rr & ir & شعان \\
\hline דזים & IV & $1 \leqslant Y v$ & rY. & 01. & 971 & ๕ & 19 & \\
\hline$r \leqslant \wedge$ & qזr & Ү११४ & OAYO & ז.. & ivaqr & 7.9 & זrז؛ & \\
\hline $1 \ldots 07$ & $1.1 \mathrm{~A}$ & 00.1. & $0 \leq 7 r 4$ & 01990 & 77791 & Trol & \$Y०१ & 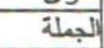 \\
\hline
\end{tabular}

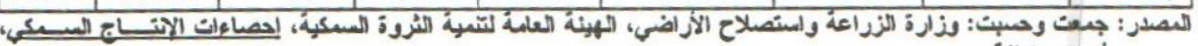

أعادل مختلفة.

أولا: أسطول الصبد:

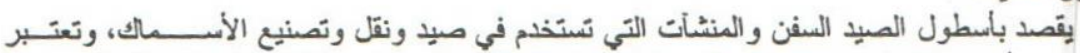

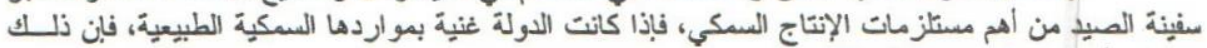
لا يعني أن لديها ثُروة سمكية هائلة.

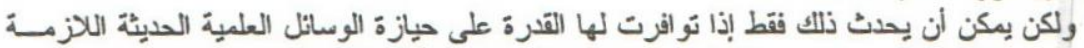

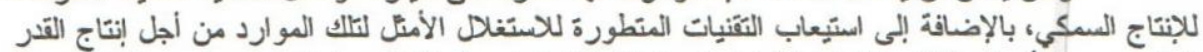

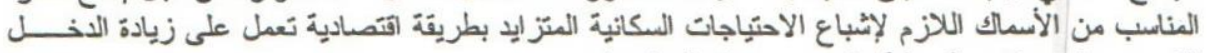

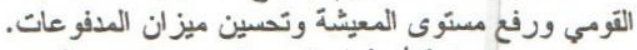

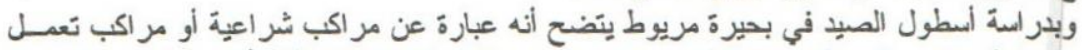

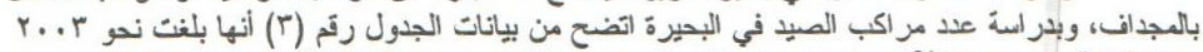

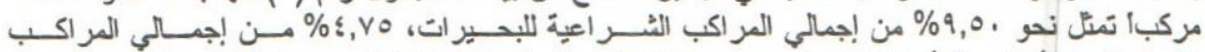

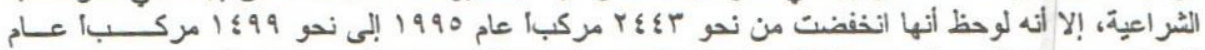

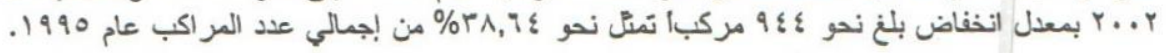

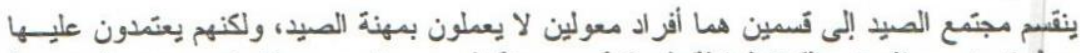
ثاتبا: عملة الصصيد:

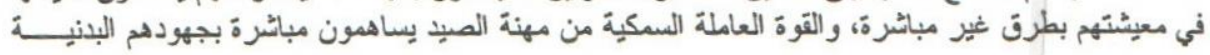




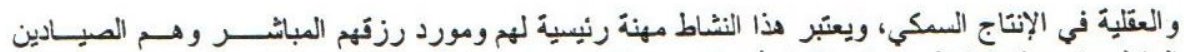

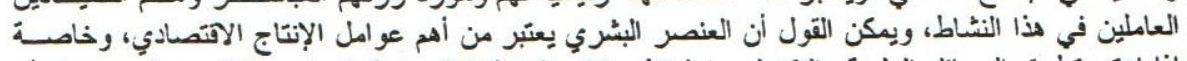

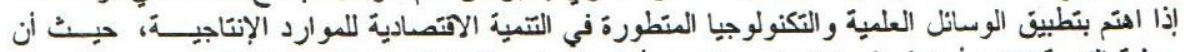

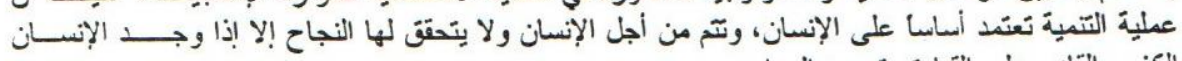

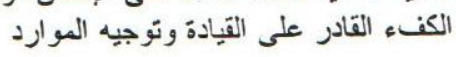

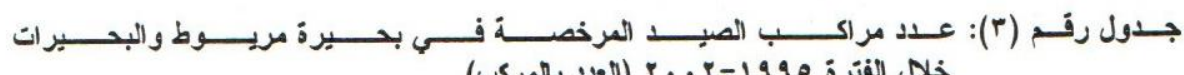

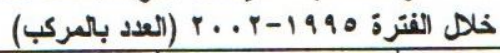

\begin{tabular}{|c|c|c|c|c|c|c|c|c|c|c|c|c|}
\hline \multicolumn{3}{|c|}{$\%$} & \multicolumn{3}{|c|}{ الجهورية } & \multicolumn{3}{|c|}{ البحيرات } & \multicolumn{3}{|c|}{ بحيرة مريوط } & \\
\hline المي وشريرية الجية & "جرهيورية' & شبراعير & الإجملى & شراعي & ألى & |الإجسلى & شراعى & ألى & الإجسلم & شراعي & ألى & \\
\hline i,V & $r, r$ & $1 \xi, r$ & ris. & rrTYo & P.r9 & 18111 & IVIII & - & $T \leq \leqslant$ & $r \leq \leqslant T$ & - & 1990 \\
\hline ؛,Y & 0,00 & $9, \Lambda$ & ז'T & ؛ AYY. & rrs? & ro.rt & ro.ri & - & $Y \leqq 0 \wedge$ & $r \leq 0 \wedge$ & - & 1997 \\
\hline$؛, V$ & $0, \cdot €$ & $1 \cdot, 1$ & oriar & $\Leftrightarrow$ ^Yฯ & $r \leqslant 10$ & rrAO. & rrAo. & - & $r \leqq 0 \wedge$ & $r \leq 0 \wedge$ & - & $199 \mathrm{~V}$ \\
\hline$\lfloor .1$ & $0,1 \leq$ & $1 \cdot, 4$ & ד.97 & ؛ VAII & rioo & אידוTr & rrTYr & - & $r \leqslant 0 \wedge$ & $r \leq 0 \wedge$ & - & 1991 \\
\hline$\{, 0$ & 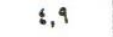 & 9,9 & rqiT! & rirvi & rYOA & IA $\leqslant r T$ & IASTT & - & IVYV & IVYV & - & 1999 \\
\hline$r, i$ & $r, r$ & $v, r$ & $\$ 0.70$ & \&.NTY & ؛rrq & riqor & riqor & - & ITI & & - & . \\
\hline$r, q$ & $r, r$ & 7,0 & ؛६११. & $\$ .907$ & rq०६ & r.ITr & r.וTr & - & IrIA & $|r| A$ & - & r.. \\
\hline$r, !$ & $r, V$ & $v, i$ & \$ะ191 & \&.rvq & rAIr & 197.5 & 197.6 & - & $1 \leqslant 99$ & $1: 99$ & - & $r \ldots r$ \\
\hline ؛. & $\Leftrightarrow, V_{0}$ & 9,0 & \$OTYV & sYlor & ror! & 81.90 & 91.90 & & $r \ldots r$ & $r \ldots r$ & & لجمة \\
\hline
\end{tabular}

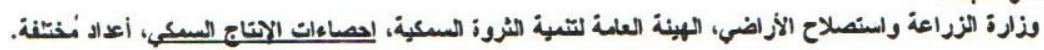

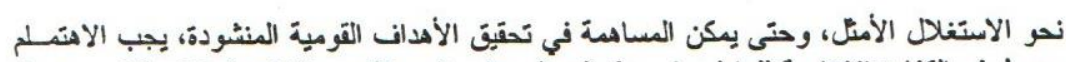

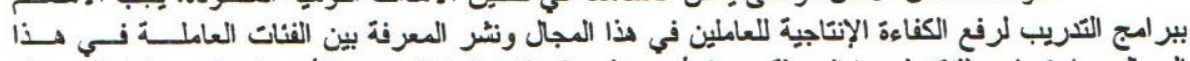

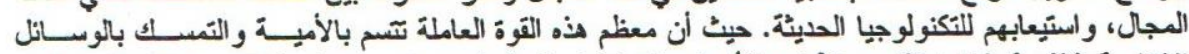

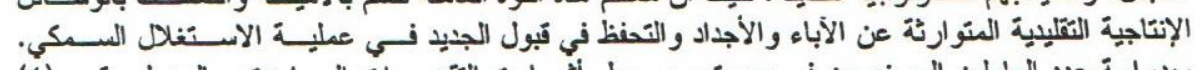

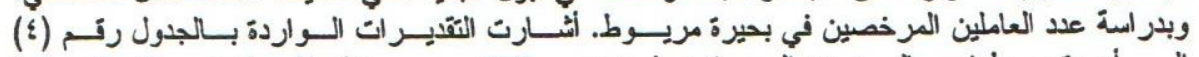

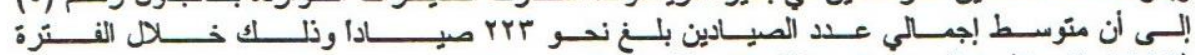

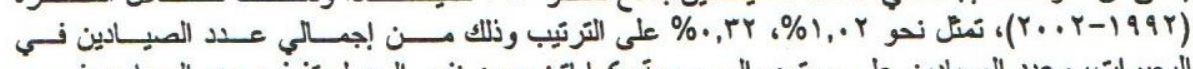

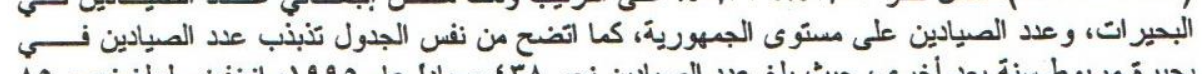

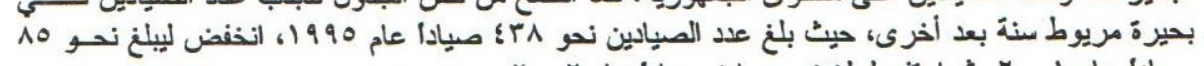

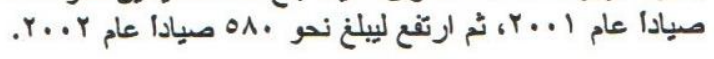

ناثةا: الإنتاج السمكي في بحيزة مريوط:

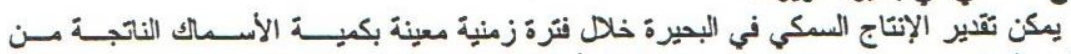

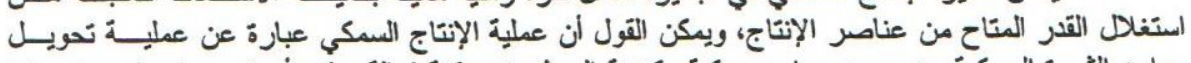

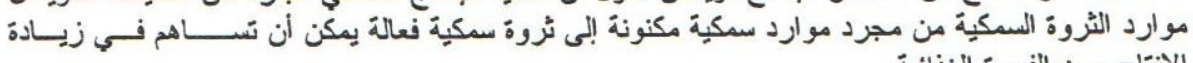
الإنتاج وسد الفجوة الغذائية.

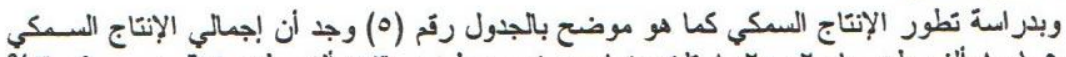

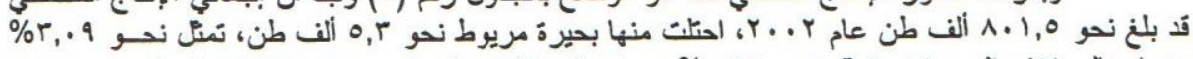

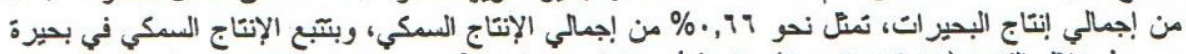

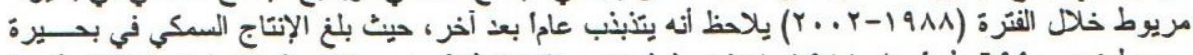

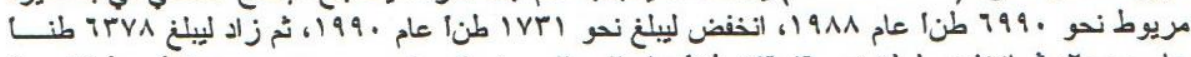

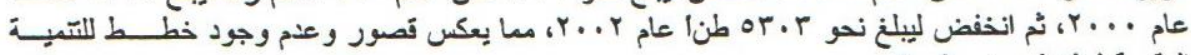
المقدمة لهذا المصدر المبكي. 
Abdel-Hkim, N. F. et al.

جدول رقم (؛) :الأهمبة النسبية لعد الصيادين المرخصين في بحيرة مريوط بالنسبة للبحيرات والجمهريـة

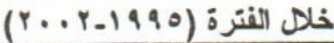

\begin{tabular}{|c|c|c|c|c|c|}
\hline \multirow[t]{2}{*}{ الجمهورية } & \multicolumn{2}{|c|}{ البحيرات } & \multicolumn{2}{|c|}{ عدد الصسادين } & \multirow[t]{2}{*}{ |لبينان } \\
\hline & & الجمهورية' & البحير ات & بحيرة مريوط & \\
\hline$\cdot, \pi$ & 1,90 & VINIY & $T Y \leq \leqslant$. & $\leqslant r \wedge$ & 1990 \\
\hline$\cdot, r \cdot$ & $\cdot, 0 \wedge$ & inror & rriog & IrV & 1997 \\
\hline$\cdot, 19$ & זוה, & iTrar & I $98 \mathrm{VV}$ & $1 Y \leq$ & $199 v$ \\
\hline$\cdot, Y r$ & rit & דירזי & rVT & $18 \leq$ & 1991 \\
\hline$\cdot, 1 T$ & $\cdot, \S 1$ & $9 . r 9 r$ & TTA.T & 1.9 & 1999 \\
\hline$\cdot, 19$ & $\cdot,()_{1}$ & ทงTा9 & IAVTY & ITr & $r \ldots$ \\
\hline$\cdot, 15$ & $\cdot, \leqslant 1$ & $7 \leq 9 \leq 7$ & $r .9 .0$ & 10 & $r \ldots l$ \\
\hline $1, \cdot v$ & $£, 9$. & $0 \leqslant \leqslant \leqslant$ & 18179 & $0 \wedge$. & $r \ldots r$ \\
\hline$\cdot, r r$ & $1, \cdot r$ & V.rVo & PIVT० & YrP,O. & هنوسط الفترزة \\
\hline
\end{tabular}

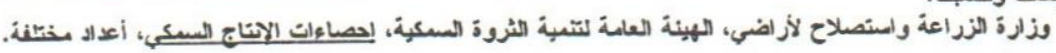

جدول رقم (0): تطور إنتاج الأسماك في بحيرة مريوط والبحيرات والجمهورية خـــله الفــرة (1991 ا-

\begin{tabular}{|c|c|c|c|c|c|}
\hline \multirow[t]{2}{*}{ الجمهورية } & \multirow[t]{2}{*}{ أبحيز ات } & \multicolumn{3}{|c|}{ الإنتاج السمكي بالطن } & \multirow[b]{2}{*}{ المسنوات } \\
\hline & & الجمهريية & البحيرات & بحيرة مريوط & \\
\hline$r, 17$ & $\varepsilon, Y Y$ & rrro.. & $1 \leqslant \wedge \ldots$ & 799. & $191 \mathrm{~N}$ \\
\hline $1, \cdot 9$ & $r, \wedge$ & $r Y \leqslant V .$. & Irvv.. & $r 0 \leqslant T$ & 1919 \\
\hline., 01 & $1, \cdot 1$ & rrva.. & 17.1. & $|V T|$ & 199. \\
\hline., $0 \mathrm{~V}$ & $1, Y 1$ & $r \leqslant r r .$. & $1711 \ldots$ & 1907 & 1991 \\
\hline$\cdot, 91$ & $1,9 v$ & $r \leq 7 \leq \ldots$ & $1091 \ldots$ & $r \mid \leqslant 1$ & 1997 \\
\hline $1, r$ & $r, r \varepsilon$ & rotvrt & lovrio & rint & 1997 \\
\hline .990 & $r, 10$ & rฟAIAะ & irgs & roli & $199 \leqslant$ \\
\hline., 10 & 1,14 & \&.V.rY & $1 \wedge \div \leqslant 4$. & $r \leqslant 7 \%$ & 1990 \\
\hline$\cdot 99$ & $r, r_{0}$ & $\varepsilon r \mid 7 \varepsilon r$ & IvTorv & rqvi & 1997 \\
\hline$\cdot, 91$ & $r, Y q$ & $\varepsilon O V \cdot r\rceil$ & $1907 \mathrm{rr}$ & $\leq \leqslant \wedge 9$ & 199v \\
\hline$\cdot, \lambda r$ & $r, I r$ & $0 \leqslant 009 r$ & rIYAAq & $\{0 Y 1$ & $199 \mathrm{~N}$ \\
\hline$\cdot, \wedge 1$ & $Y, \Lambda I$ & $7 \leq \wedge 9 \Gamma q$ & IATrRA & orro & 1999 \\
\hline$\cdot, \wedge \wedge$ & $r, i n$ & $V Y \leq \varepsilon \cdot V$ & IVTIRE & irva & $r \ldots$ \\
\hline$\cdot, \Lambda$. & $r, r \varepsilon$ & VV1010 & |10T9| & Y... & $r \cdot \cdots$ \\
\hline$\cdot, 77$ & $r, \cdot 9$ & $1.1 \leq 77$ & $|\vee| \wedge \mid$. & or.r & $r \cdots r$ \\
\hline$\cdot, \wedge 9$ & $P, \& 9$ & हY9YIV & 181879 & 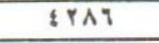 & متوسط الفترة \\
\hline
\end{tabular}

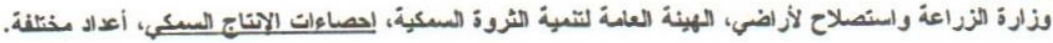

\section{التركيب النوعي للأسماك في بحيرة مريوط:}

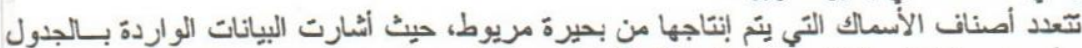

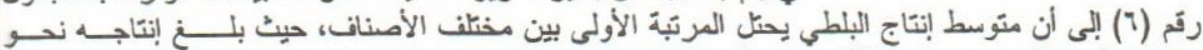

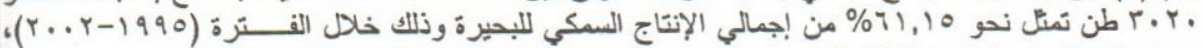

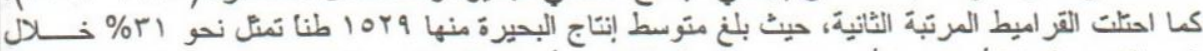

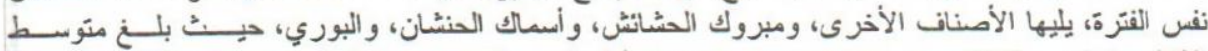

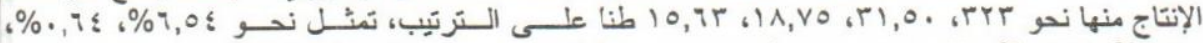

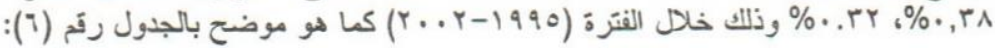




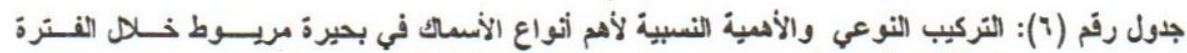

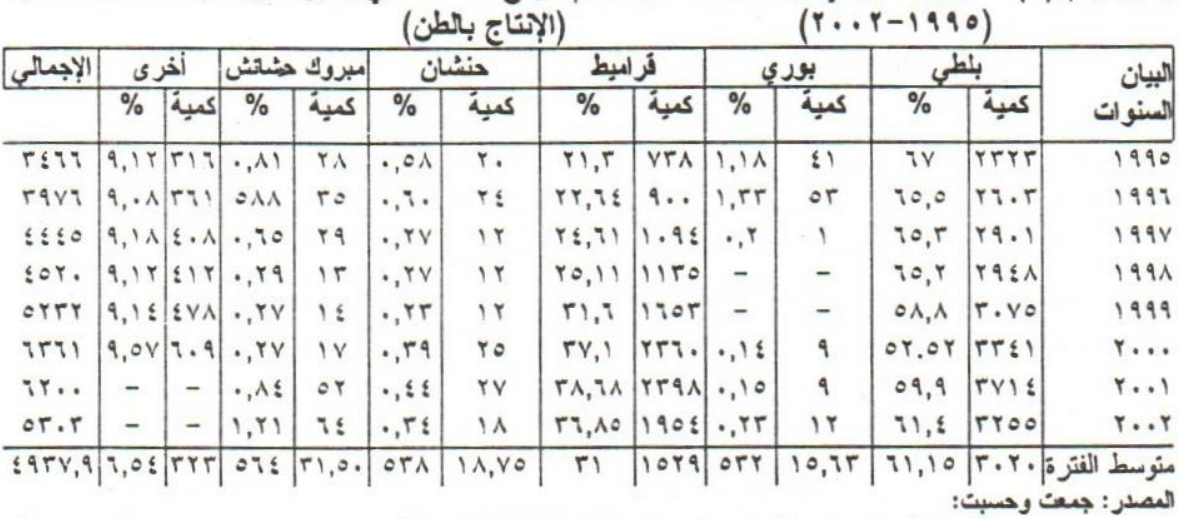

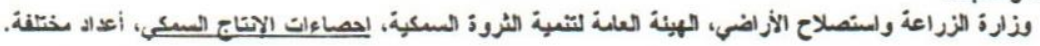

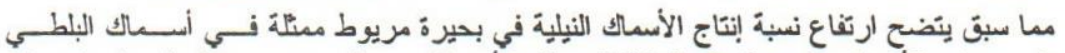

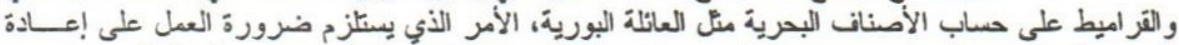

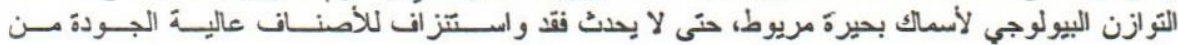

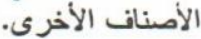

التوازن البيولوجي لأسماف بحيزة مريوط:

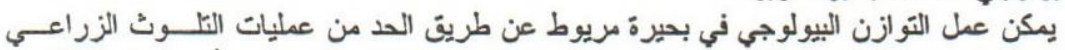

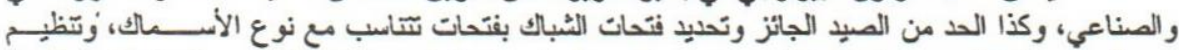

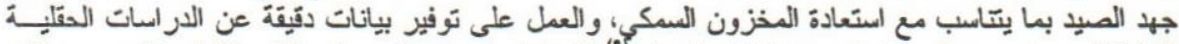

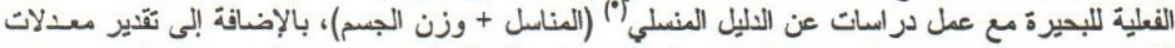

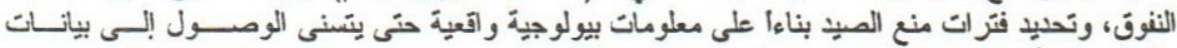

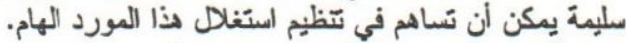

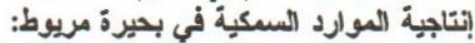

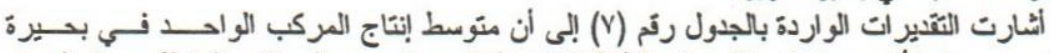

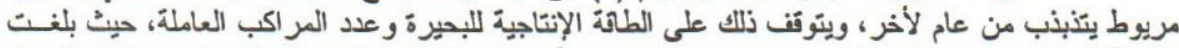

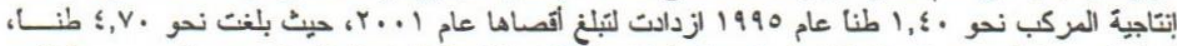

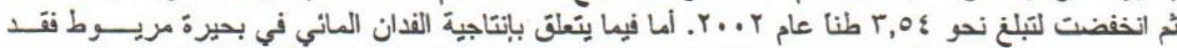

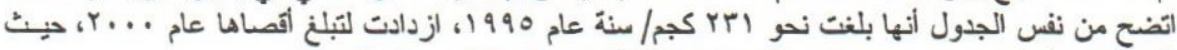

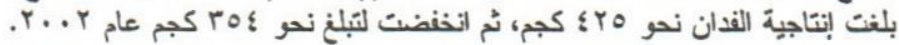

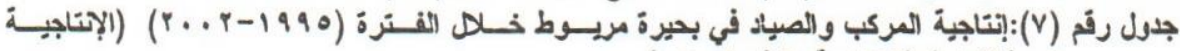

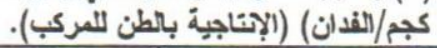

\begin{tabular}{|c|c|c|}
\hline الإتتاجية بلطن للمركب & الإنتاجية بلطن للفدان & لالمنوات \\
\hline $1, \ldots$ & rTI & 1990 \\
\hline $1, \mathrm{v1}$ & rio & 1997 \\
\hline $1, \hat{A T}$ & r99 & 1994 \\
\hline $1, \wedge r$ & $r .1$ & 1994 \\
\hline 7,90 & $r \leqslant 0$ & 1999 \\
\hline 7,99 & sto & $r \ldots$ \\
\hline$\leqq, \vee$. & $\$$ & $r \ldots 1$ \\
\hline$T, 0 \leq$ & ros & $r \ldots r$ \\
\hline T.Yo & riq & متوسط الفترة \\
\hline
\end{tabular}


Abdel-Hkim, N. F. et al.

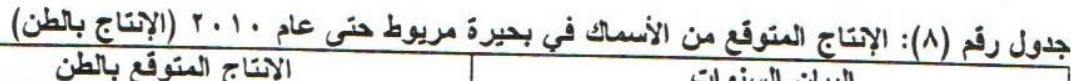

\begin{tabular}{|c|c|}
\hline الإنتاج المنتوقع بالطن & البيان السنوات \\
\hline 07.9 & $r \ldots$ \\
\hline ovvi & $r \ldots T$ \\
\hline $09 \leqslant Y$ & $r \ldots v$ \\
\hline 71.9 & $r \ldots \Lambda$ \\
\hline TrVi & $r \ldots q$ \\
\hline$T \leqslant \leqslant$ & Y.1. \\
\hline
\end{tabular}

السصدر: جعت وحسبت: من بيتات الجدول رئم (0).

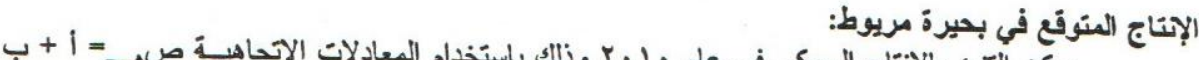

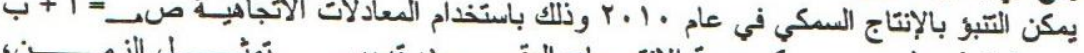

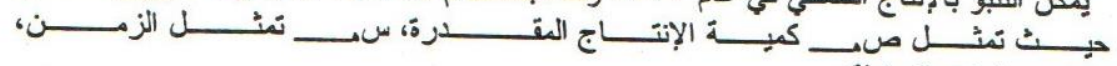

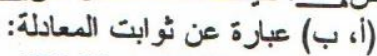
صـ $\cdot(r, \cdot 1)$ $\cdot, \leqslant 9=$ $\cdot, r \leqslant=r$ ر $\cdot(\xi, \cdot Y)=$

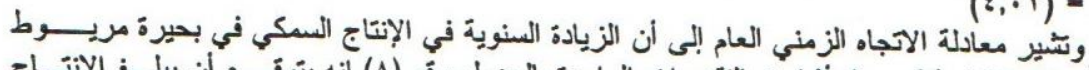

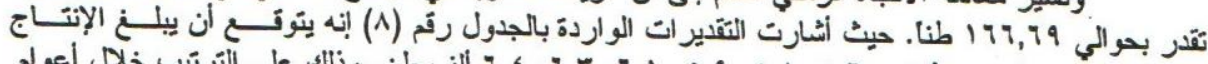

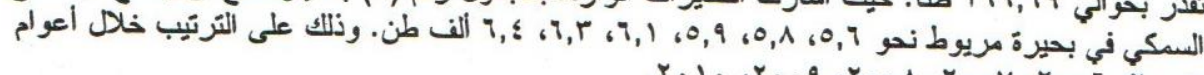

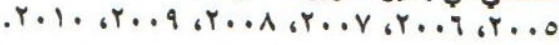

محددات الإختاج السكي في بحيرة مريوط:

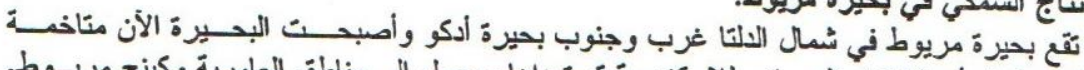

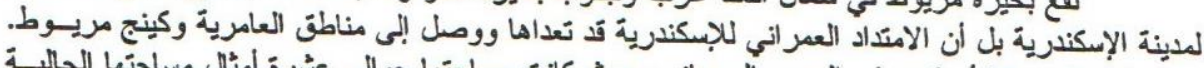

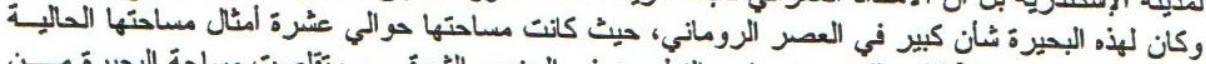

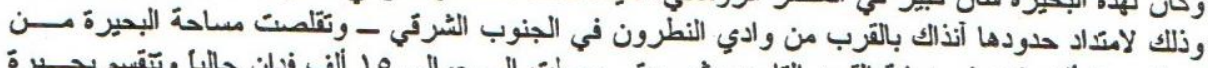

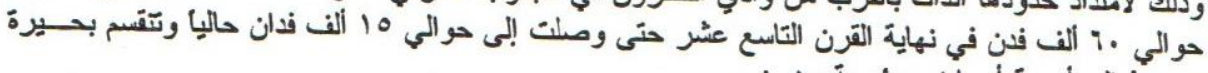

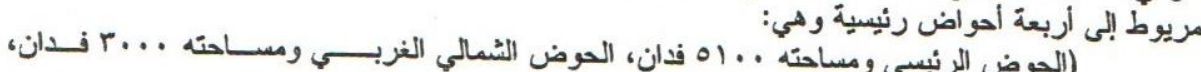

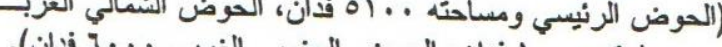

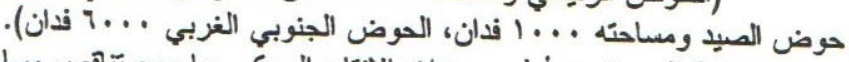

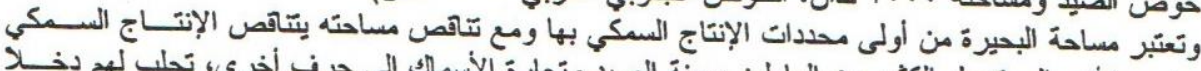

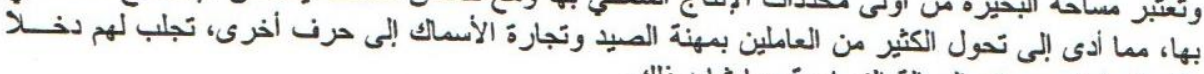

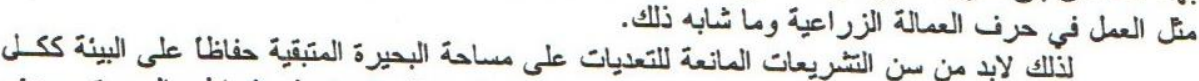

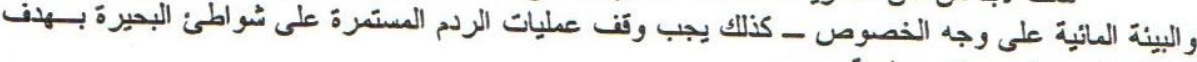

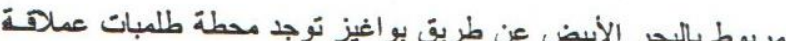

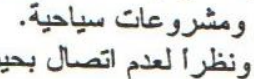

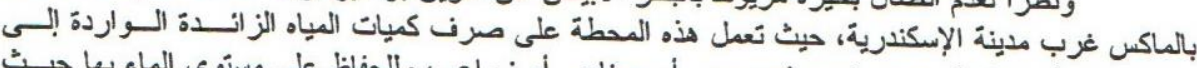

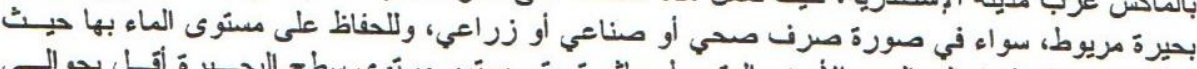

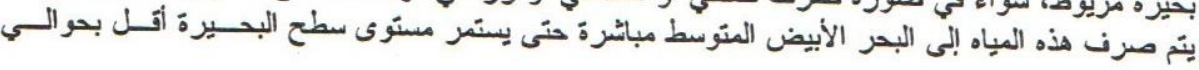




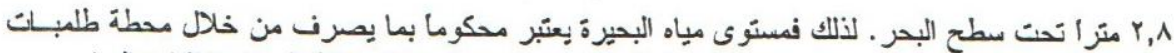

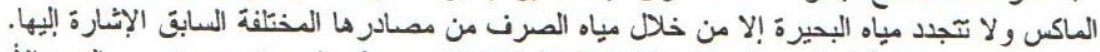

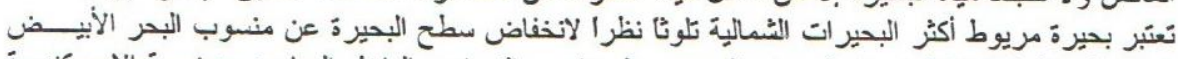

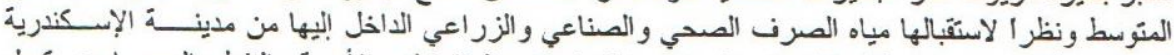

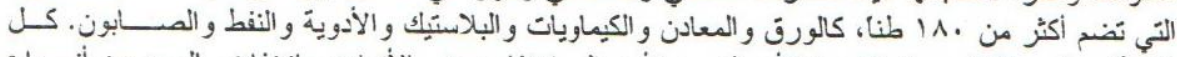

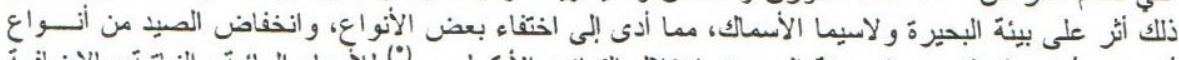

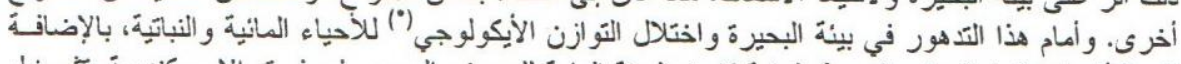

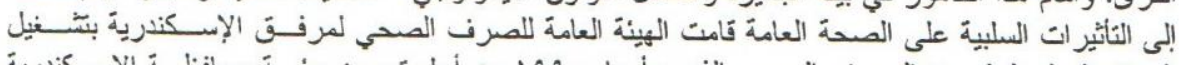

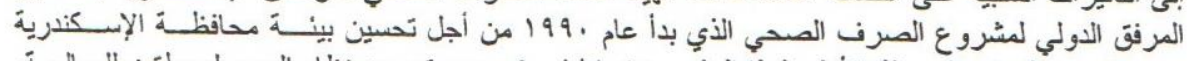

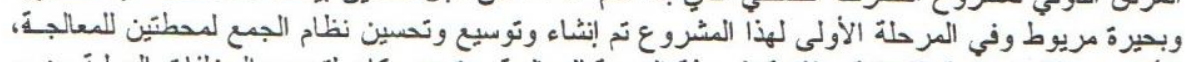

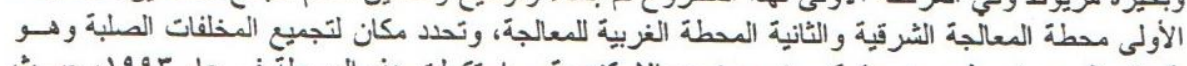

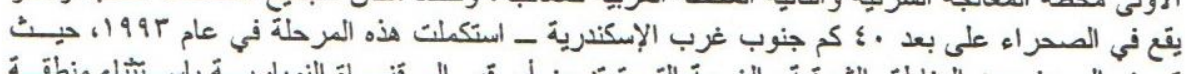

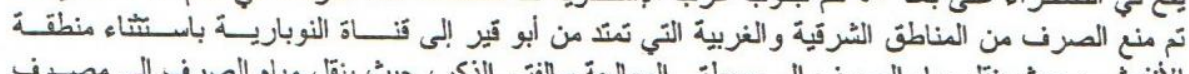

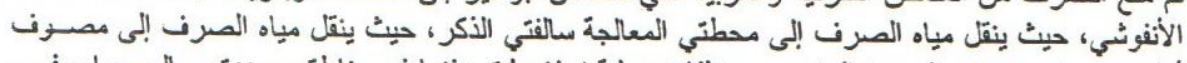

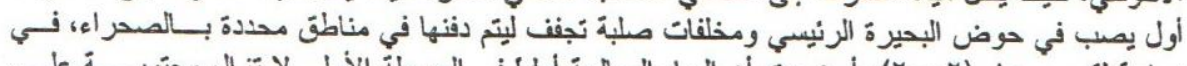

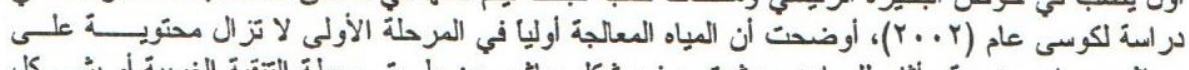

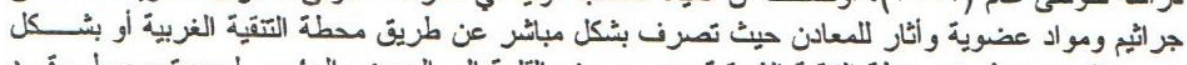

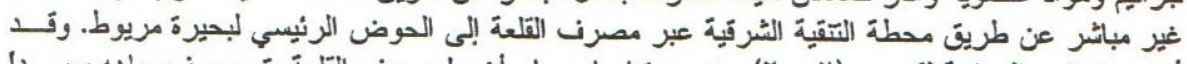

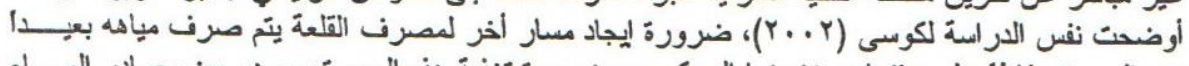

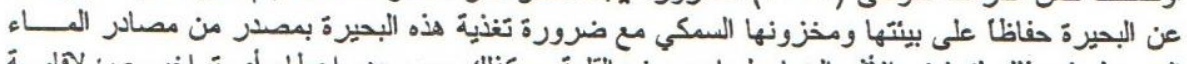

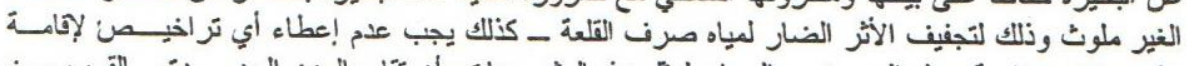

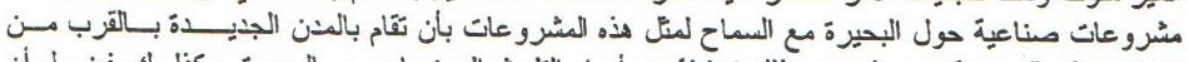

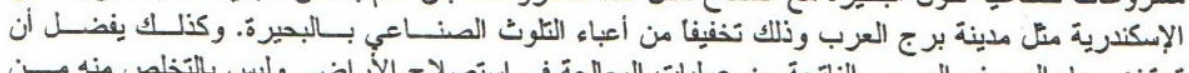

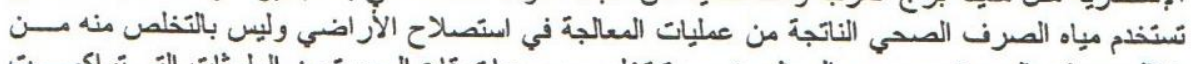

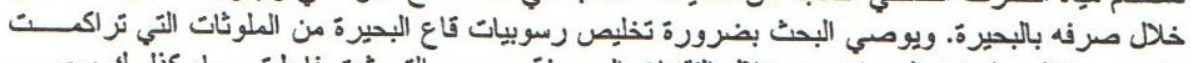

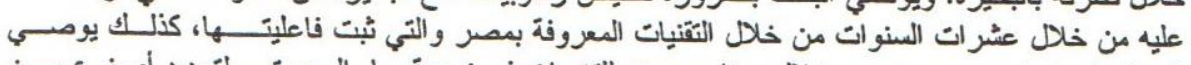

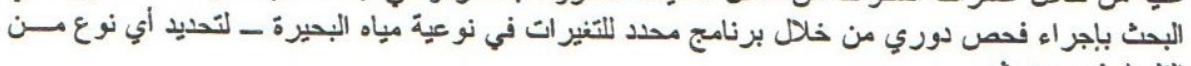

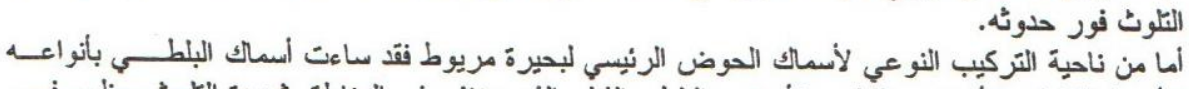

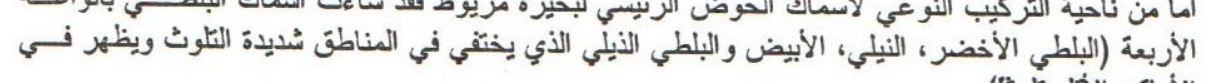

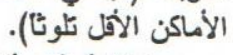

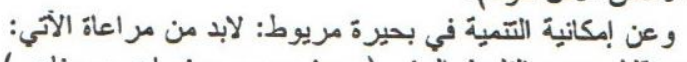

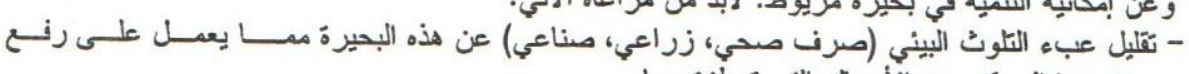

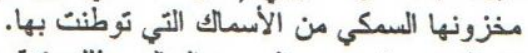

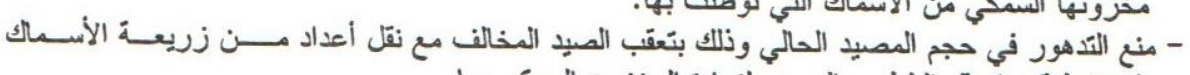

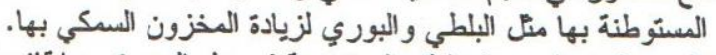

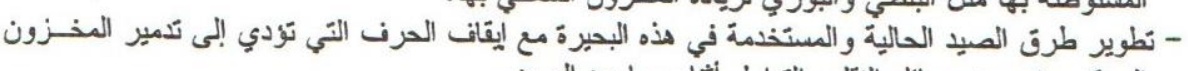

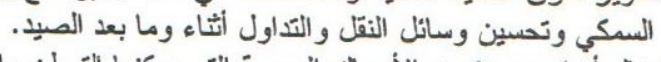

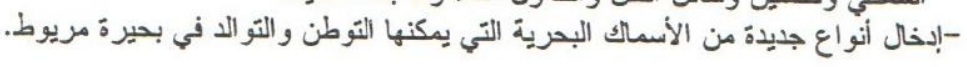




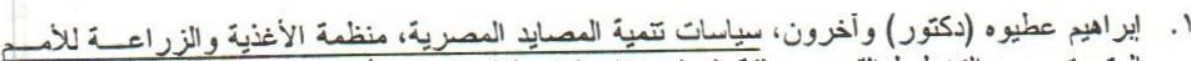

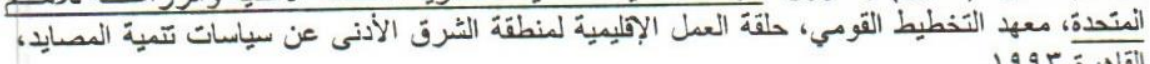

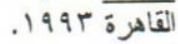

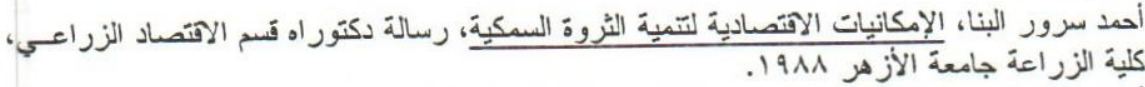

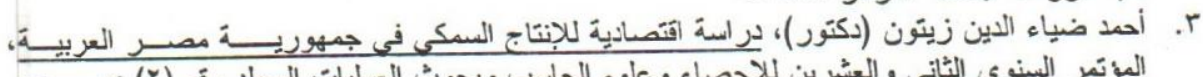

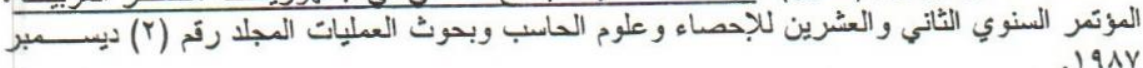

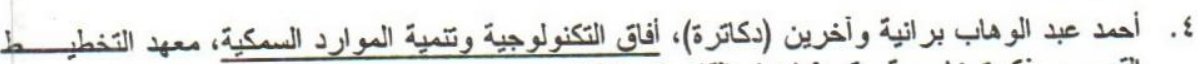

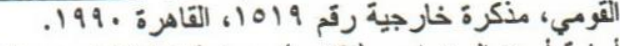

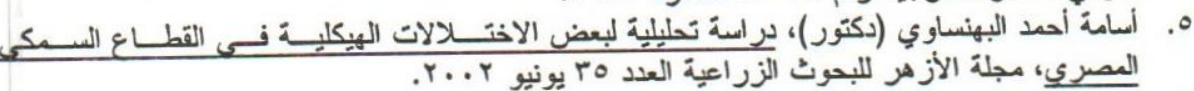

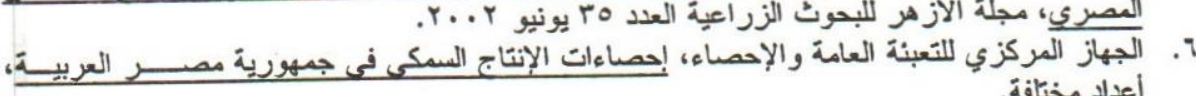

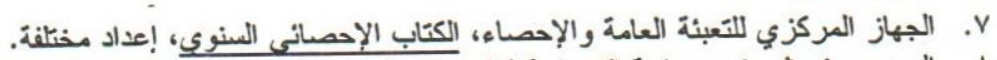

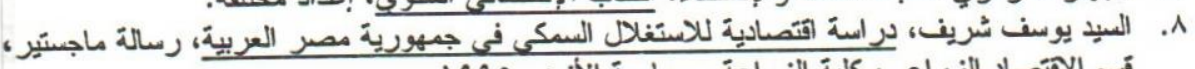

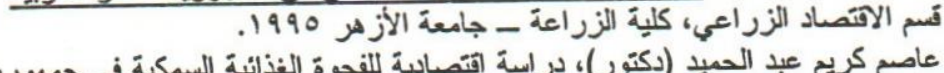

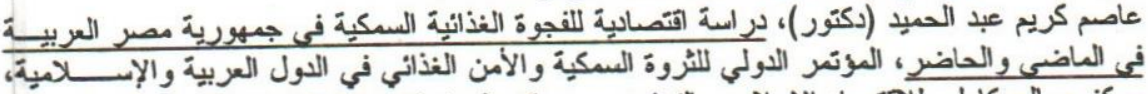

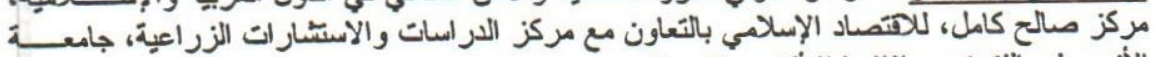

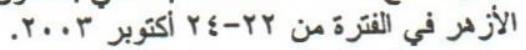

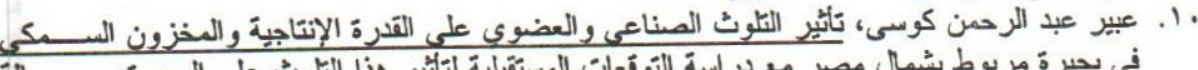

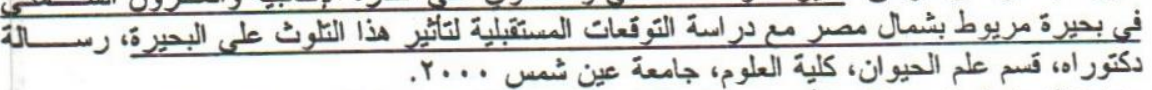

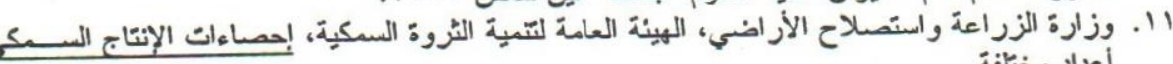

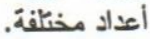

\section{ECONOMICS OF FISH PRODUCTION IN MARRIOTT LAKE Abdel-Hkim,N.F.*; A. K. Abd- Elhmied ${ }^{\star \star}$ and Sh. A.Abdel-Momen ${ }^{\star *}$ Dept. of Animal Production, Faculty of Agriculture, Al-Azhar University \\ ** Dept. of Agric. Economic production - Faculty of Agriculture-Al- Azhar University}

\section{ABCTRACT}

There are some directions towards drying the internal lakes for agricultural production purposes, in spite of their importance as main sources for fish production which led to fish nutritive gab which increases yearly., so, to overcome this problem we had to compensate this shortage by import, which led to a problem, with two branches: first is the foreign currency, the second which is more important, how to keep a minimum of national production. 
That is will be achieved by following a correct scientific management of available water sources.

Marriott's lake is one of the most important fish production lakes, but we noticed a gradual decreasing in its area from 60 thousand feddan in 1965 to approximately 15 thousand feddan in our present time, moreover, it is very polluted, which reflected negatively, on the lake's environment, and affected negatively on it's fish production.

As a result of that, some kind of fish had been disappeared and other kinds had decreased; we see clearly that in year 2002, the production of Marriott's lake from all kinds offish was 5303 ton which represented $3.13 \%$ from the total production of all lakes which was 169310 ton approximately.

That indicates the low rate of sharing of the lake's fish production. That is due to it's pollution, where had been decreased from 23 ton (19971999) decreased to 10 ton through (2000-2002)., and to avoid that decreasing we have to apply the biological balance in the lake, that will avoid loosing of high quality fish kinds.

In the same time we have to reduce pollution, stopping unfair fishing, fishing - net holes to be limited, organizing the fishing efforts. Organizing of non-fishing periods according to Biological and realistic information to get correct data which shares in using of the important sources.

In addition to that we have to issue some legislation's which protect the whole lake area from transgressions, that's will keep the whole environment, specially the water environment.

Also, we must stop filling-up of lake's shores by establishing of tourism projects.

-Its necessary to find an other direction for Al-Alkah's drrain to pour its water far from the lake to keep its environment and its stored same time we have to feed the lake by a new un-polluted water to reduce the harmful effect of Al-Alkah water.

-No more licenses for artificial projects around the lake.

-Purify the bottom of the lake from pollution which accumulated through many years.

Through this study, its clear that the fishing fleet in Marriott's lake is consists of just sail- boats or paddle boats, they are about 2003 boats which represents $9.50 \%$ from the total number of sail-boat in all lakes, and about $4.75 \%$ from the whole number of sail- boats all over the republic, through the overage period (1995-2000).

Its clear that the fishers number in Marriott's lake was about 223 fishers, which represents abort $1.02 \%, 0.32 \%$ from the whole number of fishers in all lakes and all over the republic in the same period. 